WELLBEING SERIES

\title{
THE PATIENT IS READY FOR YOU...BUT ARE YOU READY FOR THE PATIENT? THE HEALTH STATUS OF PARAMEDICS AND ITS EFFECT ON JOB PERFORMANCE
} Alexander (Sandy) MacQuarrie PhD MBA ${ }^{1^{*}}$

Paramedic Health Researcher, Senior Lecturer in Paramedicine

*s.macquarrie@griffith.edu.au

\section{OPEN ACCESS ARTICLE}

Recommended Citation: MacQuarrie, A. Wellbeing Series: The patient is ready for you... but are you ready for the patient? The health status of paramedics and its effect on job performance. Irish Journal of Paramedicine. 4(2) Dec 2019. https://doi.org/10.32378/ijp.v4i2.208

Received: 24 May 2019

Published: 5 Sep 2019

Copyright: $\odot 2019$, the authors. This is an Open Access article distributed under the terms of the Creative Commons Attribution-Non-CommercialShare Alike 4.0 International licence which permits use, distribution, and reproduction in any medium, provided the original work and any attributes thereof are properly cited, are distributed under the same licence, and that the work is not used for commercial purposes.

\section{(c) (1) (2)}

Funding/support: This information was presented at the ICoP Wellbeing Symposium in UCC, Cork on May 11. AMQ received a speaker honorarium for attendance.

\section{Competing interests: None declared}

Provenance and review: Commissioned, not peer-reviewed.

\section{Author affiliations}

1. Griffith University, Australia

\section{Abstract}

\section{Background}

In this wellbeing series we present practical advice for prehospital care providers, responders, and other shift workers. These articles are produced by experts in their field. Many of these topics were presented at the Irish College of Paramedics Wellbeing Symposium in University College Cork in May 2019.

Keywords: paramedic, wellbeing, prehospital, health. 
This presentation was primarily focused on research conducted on paramedics working for New South Wales Ambulance in Australia. It was based on three studies: a health survey, an observational study of how paramedics work and an experiment to see how "fit" they were.

This really started as in investigation into paramedic fatigue and job performance and as part of my lived experience as a paramedic. In my career I describe my health as both "good" and "bad". The literature speaks to high rates of injury and sick time, which may not be surprising and may resonate with you in your own service or practice.

After following paramedics and monitoring their physiology for about six months in the field, some common themes emerged: (1) looking at about 15 variables, there were several that significantly affected heart rate and respiratory rate on shift, (2) the strongest, most prevalent was Body Mass Index, (3) other contributors were age, posting (rural paramedics stronger response) and (4) type of call.

Remember, it is quite normal for responders to experience physiological arousal that is not linked to physical activity...think fight or flight syndrome. BMI seems to amplify this response in the paramedics studied: higher BMI meant a higher response. Remember that BMI is NOT a measure of "fatness" or even "fitness" by itself. It needs other measures. Increasing BMI is linked to an increased risk of metabolic syndrome (think Type 2 diabetes and cardiovascular disease).

On the opposite end of the scale, the "fitter" the paramedics were from an aerobic capacity standpoint, the lower their physiological response compared to unfit. The measure we used was $\mathrm{VO}_{2}$ max.

While there needs to be much, much more research, the takeaway points for each of you includes the following:

1. If your health is not optimum, understand that you, your family and your employer all have a vested interest in making it better

2. Health status is measured a lot of different ways (BMI and aerobic fitness for example).

3. To impact these, the addition/change or continuation of structured physical activity (exercise) is needed. Same for nutrition, which is so important to good health.

4. At the same time, you need the best tools to do the job (think of powered stretchers and other equipment that can reduce the potential for injury). It does not let you off the hook for no. 3 above.

In the end, the job of paramedic (or emergency responder in general) is unpredictable and the next call cannot be predicted. However, what can be affected in a positive way is your own health. Keep responding, keep supporting those who respond and keep supporting those who work to study and understand the health of responders. 